\title{
11
}

\section{The Bundle-Spacer: A Cost Effective Alternative for Traffic Shap- ing in ATM Networks ${ }^{1}$}

\author{
J. Garcia*, C. Blondia**, O. Casals*, F. Panken** \\ * Polytechnic University of Catalonia, Computer Architecture Department \\ c/ Gran Capitan, Modulo D6, E-08071 Barcelona, Spain \\ tel : + 343 4016985, fax : + 343 4017055, e-mail : olga@ac.upc.es \\ ** University of Nijmegen, Computer Science Department \\ Toernooiveld, 1, Nl-6525 Nijmegen, The Netherlands \\ tel : + 3180652590, fax : + 3180553450 , e-mail : chrisb@cs.kun.nl
}

\begin{abstract}
This paper deals with a traffic shaper which spaces all the cells belonging to a bundle of VCs, hence its name bundle-spacer. Although the concept of a bundlespacer is applicable in general to situations where no VC information is available, we have focused at one particular application, namely the access control of a shared access link to an ATM network. The dimensioning of the bundle-spacer, i.e. finding an optimal value for the spacing period, is achieved by means of a simple rule, called the Bounded Period Rule. This rule aims at finding a compromise between having an almost perfect spacing for high bit rate connections (and hence avoiding clumps of cells) while avoiding high values of delay and CDV for low bit rate connections. Using different performance measures, it is shown that this rule turns the bundlespacer into both a cost effective and efficient traffic shaping device.
\end{abstract}

\section{Introduction}

In an ATM network new calls are accepted on basis of a contract established between the user who originates the call and the network. This traffic contract consists of the source traffic descriptor (e.g. Peak Cell Rate, Sustainable Cell Rate and Burst Tolerance), the CDV (Cell Delay Variation) Tolerance and a requested Quality of Service (QoS) class which are used by Call Admission Control (CAC) to decide whether sufficient resources are available to establish the connection through the whole network at its required QoS while maintaining the aggreed QoS of existing connections.

The Usage Parameter Control (UPC) function will use this descriptor to check if the cell stream offered to the network by the accepted call is conforming to the contract. Due

\footnotetext{
${ }^{1}$ This work was supported in part by the Commission of the European Communities, under project RACE R2024 (Research and Development on Advanced Communications in Europe) on Broadband Access Facilities.
} 
to the passage through multiplexers and switching stages, the traffic characteristics of the cell stream are altered (in particular jittered), resulting in clumping and dispersion of cells. When the cell stream of a connection is altered by CDV, some tolerance has to be introduced in the policing function by the network and therefore, CDV must be part of the Traffic Contract. So conformity to the Traffic Contract at the network entry point does not imply that the traffic offered by the connection is actually conforming to the negociated peak cell rate. A "malicious user" could then send a "Worst Case Traffic" (WCT), i.e. a burst of back to back cells with length the largest compatible with the tolerance introduced in the policing function, alternating with an idle period with length the smallest compatible with the policed peak cell rate. Therefore, allocation of resources to a connection is based on WCT that can pass the policing function. This implies a decrease of network utilization to obtain a given QoS. In order to avoid the problems caused by this situation, both within the network and at the receiving terminal, the use of a spacer has been proposed (see [1],[2]). In a spacer, the cells belonging to a Virtual Circuit (VC) are stored (if necessary) and rescheduled such that the resulting cell stream is conforming to the contract. The memory needed to deal with the high number of VCs and the speed at which it has to operate, turn such a spacer into a complex and expensive network device. In the sequel, we refer to this spacer as a VC-spacer.

In this paper, we propose an alternative to the above spacer, called a bundle-spacer. Such a device spaces all the cells belonging to a bundle of VCs (typical a Virtual Path (VP)). In general a bundle-spacer may be applied in situations where traffic shaping is needed and no $\mathrm{VC}$ information is available (because this information is not known or because it is too expensive to use it). Let us give two application areas for a bundle-spacer. A bundle-spacer used in an ATM access network.

In [3], a MAC protocol has been proposed to concentrate the traffic originating from different T-interfaces (NT) onto a single network access link (see figure 1). The central controller (located at the Line Termination (LT)) distributes the available bandwidth of the shared link among the different NTs. The LT must issue a permit addressed to a given NT to allow this NT to send a cell. The bandwidth is distributed based on the information received from the NTs by means of requests, which are issued from time to time. Ideally, the requests should contain the arrival instant of each cell, but due to the small overhead available for these requests, an NT can just send information about the number of cell arrivals since the last request that was issued by this NT. If more than one cell has arrived at an NT since its last request was issued, then the LT will receive a request for several permits. In order to avoid clumps of cells originating from the same NT, the controller enforces a distance between consecutive permits addressed to a given NT (i.e. consecutive cells are spaced). As the LT has only knowledge about the bandwidth requirements of NTs (and not about the individual VCs carried by the NTs), this shaping function can be seen as a bundle-spacer. In this case the bundle consists of all VCs carried by an NT. A VP-spacer

In a VP-based ATM network, VCs are grouped into VPs. Assume that we have $\mathrm{N}$ sources which make up the traffic of the output VPC in the private network (figure $2 \mathrm{a}$ ). The peak bit rate of this VP $\left(\lambda_{V P}\right)$ is the sum of the peak bit rate of the sources. At the $T_{B}$ interface we have a UPC mechanism which has to be dimensioned according to this peak bit rate. Credits are generated at a rate $\lambda_{V P}(1+\tau)$, where $\tau$ is a certain tolerance which 


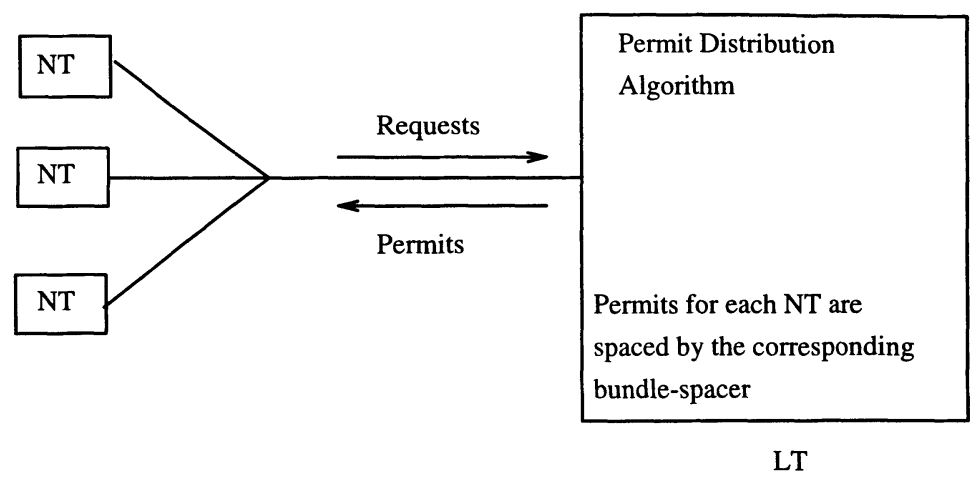

Figure 1: ATM access network

has to be determined. The problem is that the dimensioning of the UPC (i.e. finding an appropriate value for $\tau$ ) depends mainly on the way the sources are polled. Consider two sources which fill the VP. One emits one cell every 10 slots and the other one emits one cell every 40 slots. Assume now that the sources are polled at the rate of the slowest source (figure $2 \mathrm{~b}$ ), this means that at the $T_{B}$ interface we have clumps of cells at the full link rate. Therefore the UPC should generate credits at the link rate if it does not want to discard cells emitted conforming to the contract! A possible solution to this problem would be to use a VP-spacer before the UPC which should enforce a certain distance between cells.

Clearly, a bundle-spacer may offer a cost-effective alternative to a VC-spacer, provided

(i) it fulfills its basic function, i.e. protects the QoS of the users, avoiding clumps of cells originating from the same bundle of VCs

(ii) the waiting time introduced by the bundle-spacer on the cells of individual VCs is limited

(iii) it does not introduce unacceptable delay variations (CDV) on the cells of individual $\mathrm{VCs}$

(iv) it can be dimensioned such that the above conditions are satisfied.

The aim of this paper is to investigate the above characteristics of a bundle-spacer, using the appropriate performance measures and models. We focus on the first application of the bundle-spacer, namely a bundle-spacer used in an ATM access network.

In Section 2, we describe the characteristics of a bundle-spacer. Section 3 studies the traffic characteristics of individual VCs at the output of the bundle-spacer, using two performance measures : the coefficient of variation of the interdeparture time and the loss probability in a Virtual Leaky Bucket. In Section 4 a simple algorithm, called the Bounded Period Rule (BPR), for determining the spacing period is proposed. The efficiency of the bundle-spacer is studied for both well-behaving customers and worst-case 


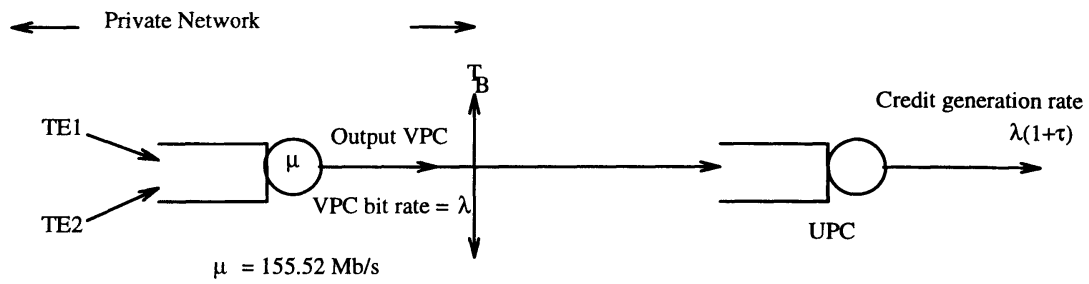

Figure 2a

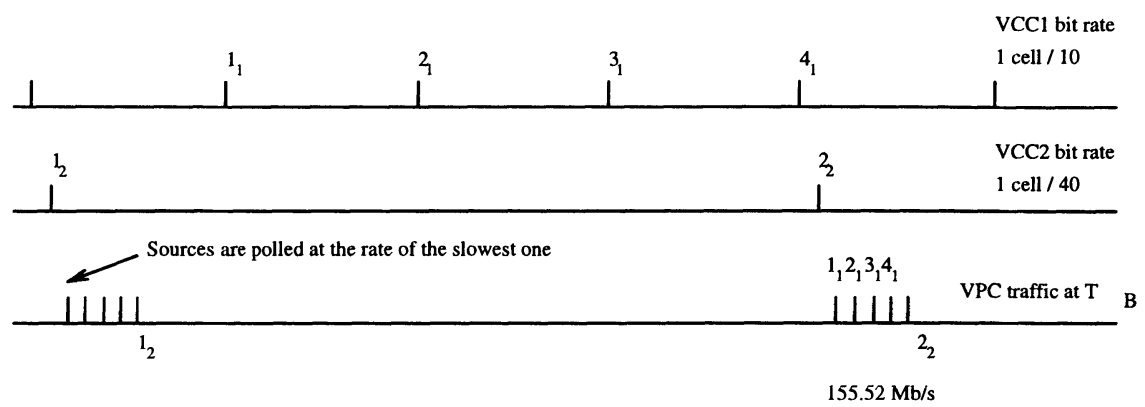

Figure 2b

Figure 2: A VP-based ATM network

traffic customers. In Section 5 conclusions are drawn and directions for further research are given.

\section{Characteristics of a Bundle-Spacer}

In the case of the first application described in the introduction (an ATM access network), the CDV before the bundle-spacer is due essentially to the mechanism used to collect requests. If we have just a few bits for MAC information in each time slot, we cannot have a perfect knowledge of the arrival time of each cell. This effect is specially relevant for high bit rate connections as it can introduce cell clumping. Therefore a spacing device should be introduced. By using a bundle-spacer with a spacing period equal to the inverse of the sum of the peak bit rate of the individual connections $\left(T_{s p}=1 / \lambda_{V P}\right)$, we could avoid the occurrence of consecutive cells for high bit rate connections. However, when multiplexing low bit rate connections, the bundle-spacer would introduce large delays and 
values of (absolute) CDV (see [3]).

In the case of the second application, the clumps would be introduced depending on the way the sources are polled to make up the VP. Again the use of a bundle-spacer with period $T_{s p}=1 / \lambda_{V P}$ could introduce large delays when multiplexing low bit rate connections.

Hence, when dimensioning the bundle-spacer (i.e. determine a value for the spacing period), we clearly have a trade off between

(i) the high transfer delay and CDV a spacer may introduce for low bit rate sources (therefore, it spaces as less as possible)

(ii) avoiding clumps of cells for high bit rate connections (therefore, space as strict as possible).

\section{Evaluation of the Traffic Characteristics at the Output of the Bundle-Spacer}

In the previous section we have seen that fixing the spacing period equal to the inverse of the sum of the peak cell rate of the VCs belonging to the bundle may lead to long waiting times and high values of CDV. In this section we investigate in a quantitative way how the speed of the bundle-spacer influences its output process. In particular, we tag a $\mathrm{VC}$ belonging to the bundle and study the cell stream of that $\mathrm{VC}$ after passing the bundle-spacer for variable values of the spacing period.

To this end two performance measures are introduced :

(i) the coefficient of variation of the interdeparture time of cells of the tagged VC and

(ii) the loss probability in a single server queue with deterministic server and with input the tagged VC (after it has passed the bundle-spacer). This queueing system can be regarded as a virtual leaky bucket.

\subsection{Coefficient of variation of the interdeparture time}

First we consider the squared coefficient of variation of the interdeparture time distribution of cells of the tagged VC which leave the bundle-spacer (denoted by CV). The $\mathrm{CV}$ is defined as the variance of the interdeparture time divided by the square mean of the interdeparture time and can be seen as a measure for the CDV. The analysis of the queueing model is done in appendix 1.

\section{a. Queueing Model}

We are interested in the behaviour of the $\mathrm{CV}$ as a function of the spacing period when the input of the bundle-spacer consists of both cells of a tagged VC and background cells. All background cells are assumed to arrive at the bundle-spacer according to a Poisson process with a rate $\lambda$. Consider the following traffic scenarios of the tagged VC :

- Batch Poisson process : In this situation geometrically distributed batches of tagged cells with a mean batch size of $1 /(1-b)$ arrive at the bundle-spacer according to a Poisson process with arrival rate $\lambda_{t}$. For the special parameter setting $b=0$ we have a Poisson process. 
- VBR/CBR : When a tagged cell has been generated, this source generates with probability $p$ the next cell after $d$ timeslots and with probability $1-p$ the source waits $s$ timeslots to send the next cell $(s, d \geq 1)$. We note that for specific parameter settings (namely $s=d$ ) this arrival pattern reduces to CBR traffic.

\section{b. Numerical Examples}

\section{1) Batch Poisson Process}

To increase the visual aspect of figure 3 , we divide the $\mathrm{CV}$ of each cell stream by the coefficient of variation of the original input stream, which is equal to $(1+b) /(1-b)$. In this figure the buffer capacity is infinite, the arrival rate of background cells equals $\lambda=0.01$ while the parameters $b$ and $\lambda_{t}$ are chosen such that $\rho_{t}=\rho_{b}$ (denoting respectively the load of the tagged and background traffic). The figure shows for all mean batch sizes a decreasing behaviour of the $\mathrm{CV}$ as a function of the spacing period, which indicates a decreasing CDV. From this figure we also observe that increasing the spacing period has more impact on arrival streams with large batch sizes, which indicates that bundle-spacing has more positive impact when the tagged VC-traffic is bursty. The positive impact of the bundle-spacer on a tagged $\mathrm{VC}$, however, decreases when the fraction $\rho_{t} / \rho_{b}$ becomes small $(\ll 1)$, i.e. when the relative bit rate of the $\mathrm{VC}$ is small.

\section{2) $C B R$ traffic}

From figure 4 we observe that when the tagged arrival pattern is CBR, the CV of the interdeparture time distribution shows an increasing behaviour when the spacing period is increased. This effect can be explained by noting that the coefficient of variation of a CBR input cell stream is equal to zero. When the spacing period is increased the virtual waiting time of arriving CBR cells increases, leading to a higher variation of the interdeparture process of CBR cells.

\section{3) VBR traffic}

For VBR traffic (figures 5 and 6 ) we observe that the impact of the bundle-spacer on the $\mathrm{CV}$ of a tagged VC-connection depends heavily on the length of the silent period $s$ and the switching parameter $p$. When the silent period is relatively small $(s=20,30)$ with respect to the busy period $(d=10)$, we observe that the bundle-spacer increases the CV of the tagged VC-connection when the spacing period increases. This situation corresponds with rather smooth traffic. For relative high values of the silent period $(s>50)$, corresponding with bursty traffic, we observe a decreasing behaviour of the CV as a function of the spacing period. In figure 5 the dotted curve indicates the behaviour for $p=0.8$ while the other curve denotes the situation for $p=0.4$. Increasing the switching parameter $p$ leads in the beginning $(p \leq 0.83)$ to a more bursty traffic pattern, which again leads to sooner positive impact on the $\mathrm{CV}$ of tagged cells. The phenomenon of the positive impact of the bundle-spacer on a tagged $\mathrm{VC}$ which decreases when the fraction $\rho t / \rho_{b}$ becomes small, was also experienced for VBR traffic.

\subsection{Loss probability in a virtual leaky bucket}

\section{a. A Virtual Leaky Bucket}




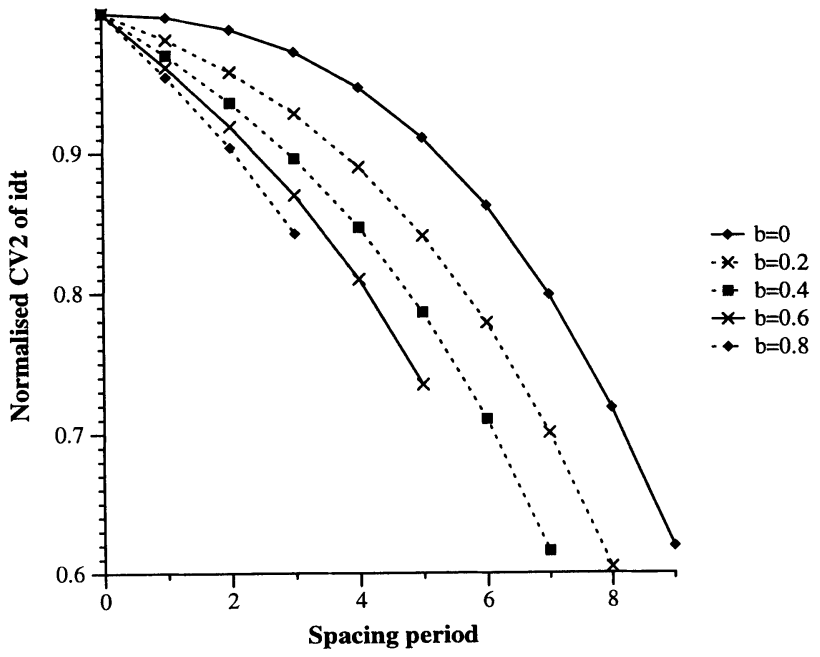

Figure 3: The CV of tagged batched Poisson cells as a function of the spacing period, with infinite buffer capacity

In order to verify that the cell stream of a VC behaves conforming to the traffic contract, we let its cells enter a finite capacity single server queue with deterministic service time and evaluate the cell loss probability. In fact, this probability equals the probability that cells are discarded when the cell stream is offered to a leaky bucket (with leak equal to the service rate and bucket size the buffer capacity of the queue). For example, if the $\mathrm{VC}$ originally is a CBR cell stream, then by letting the service time equal to the distance between consecutive cells, the loss probability is a measure for the frequency by which the peak cell rate is violated. This performance measure clearly captures the notion of clumps of cells.

\section{b. The Queueing Model}

We consider a discrete-time tandem queue consisting of the bundle-spacer and the virtual leaky bucket (VLB) (figure 7). The service time of the bundle-spacer is chosen as unit of time. The input of the spacer consists of two different cells stream :

(i) Background traffic: the number of cells arriving in one time slot is a renewal process (e.g. the background process is a Poisson process with arrival rate $\lambda$, then the probability that $n$ cells arrive during a time slot is given by $b_{n}=\frac{\lambda^{n}}{n !} e^{-\lambda}$.)

(ii) Tagged $V C$ : the interarrival time of the cells of the tagged $\mathrm{VC}$ is a renewal process, where an interarrival time of 0 is allowed (i.e. a batch of cells). Let $\bar{a}=\left(a_{0} a_{1} \ldots a_{m}\right)$ be the probability density function of the interarrival time (in slots) of cells, i.e. $a_{n}$ is the probability that the interarrival time of consecutive cells of the tagged $\mathrm{VC}$ is $n$ slots. Then clearly $\lambda_{t}=1 /\left(\sum_{i=0}^{m} i a_{i}\right)$ is the arrival rate of the tagged VC. 


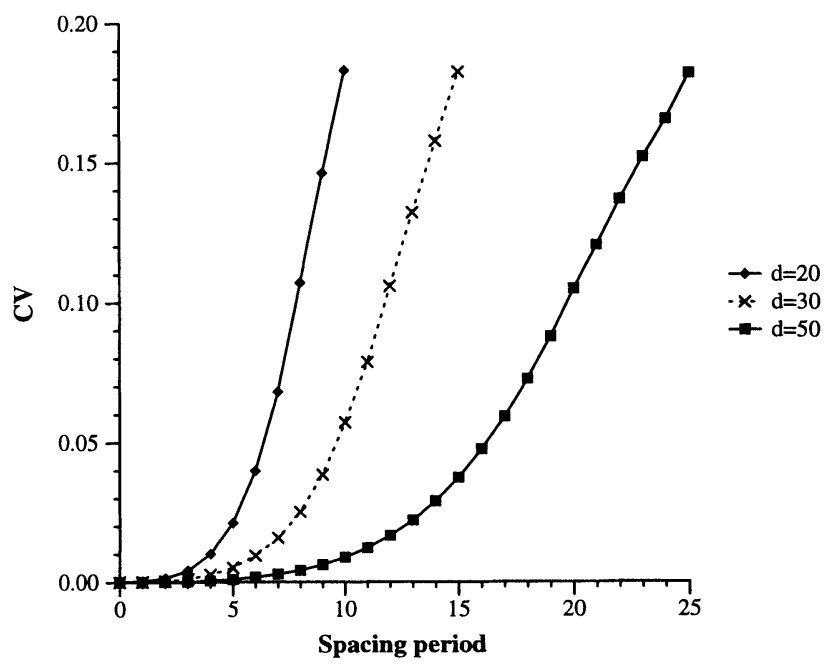

Figure 4: The CV of tagged CBR cells as a function of the spacing period. Buffer capacity of 10 .

The bundle-spacer is a single server queue with buffer capacity $N_{1}$ and deterministic service time (equal to $\mathrm{S}$ ). After having passed the bundle-spacer we let the tagged VC enter a single server queue (i.e. the virtual leaky bucket) with buffer capacity $N_{2}$ and deterministic service time $d \leq 1 / \lambda_{t}$. The background traffic is ignored.

We briefly outline how the loss probability of tagged cells in the VLB can be computed. We observe the system (i.e. the bundle-spacer and the VLB) at arrival instants of cells of the tagged VC. The behavior of the system is described by means of two variables :

- $i_{1}(n)$ : the queue length of the bundle-spacer seen by th $\mathrm{n}$-th arriving cell of the tagged VC

- $i_{1=2}(n):$ the queue length of the VLB seen by th $\mathrm{n}$-th arriving cell of the tagged $\mathrm{VC}$.

Since the number of background traffic cells is a renewal process and the interarrival time of cells of the tagged VC is also renewal, the process described by $\left(i_{1}(n), i_{2}(n)\right)$ is a Markov chain. The transitions

$$
\left(i_{1}(n), i_{2}(n)\right) \longrightarrow\left(i_{1}(n+1), i_{2}(n+1)\right)
$$

are easily derived. Once the steady state solution of this system is obtained, is is possible to compute the probability that a tagged cell arrives at a full VLB (i.e. the marginal probability that $i_{2}=N_{2}$. This results into the loss probability of cells of the tagged VC at the VLB.

c. Numerical Examples 


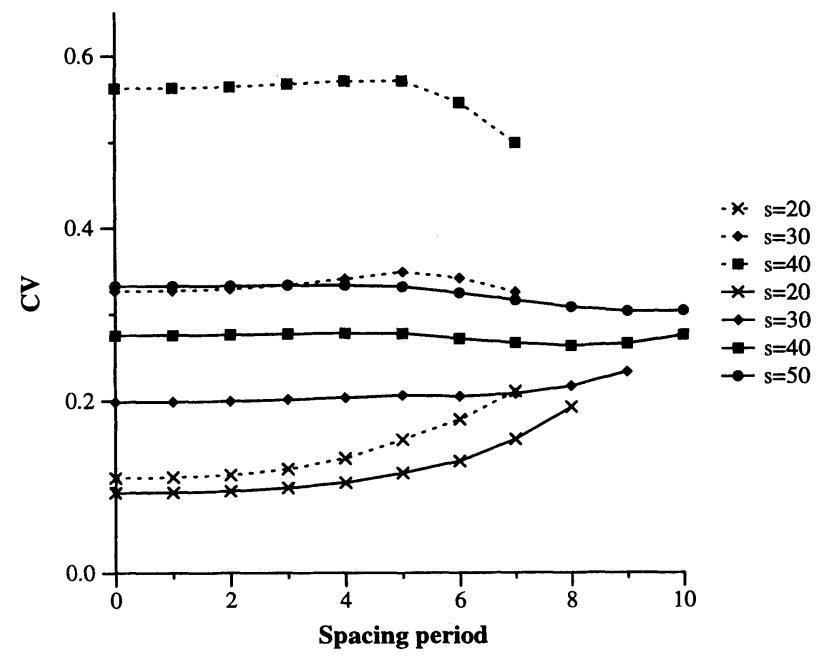

Figure 5: The CV of a tagged VBR-VC as a function of the spacing period, for different values of the silent period $s$ when $d=10$ and the buffer capacity of 10 .

\section{(i) CBR Traffic}

Consider a Poisson background traffic with arrival rate $\lambda$. We let the tagged traffic stream be a CBR source, with arrival rate $\lambda_{t}=\lambda$.

Hence, we have that $\lambda=0.5$ and that $\bar{a}=\left(\begin{array}{lll}0 & 0 & 1\end{array}\right)$. The service time of the bundle-spacer $S$ varies and the service time of the VLB is equal to the interarrival time of the tagged $\mathrm{VC}$ before the bundle-spacer. In this way, the loss probability of the VLB is a measure of how frequently the tagged $\mathrm{VC}$ violates the peak cell rate after the bundle-spacer. In the following table we show the buffer capacity $N_{2}$ needed to have a cell loss at the VLB lower than $10^{-9}$. Remark that $S=1$ corresponds to a service time equal to $1 /\left(\lambda+\lambda_{t}\right)$.

\begin{tabular}{|l|c|c|c|c|}
\hline$S$ & $S=1$ & $S=2 / 3$ & $S=0.5$ & $S=2 / 5$ \\
\hline$N_{2}$ & 3 & 2 & 1 & 1 \\
\hline
\end{tabular}

In the second example we show the corresponding results when the tagged VC is a CBR stream with arrival rate equal to $1 / 2$ of the arrival rate of the background traffic.

\begin{tabular}{|l|c|c|c|c|}
\hline$S$ & $S=1$ & $S=0.75$ & $S=0.6$ & $S=0.5$ \\
\hline$N_{2}$ & 2 & 1 & 1 & 1 \\
\hline
\end{tabular}

From the above examples we see that increasing the spacing period implies an increase of the loss probability in the VLB, meaning that the cell stream of a single CBR source may 


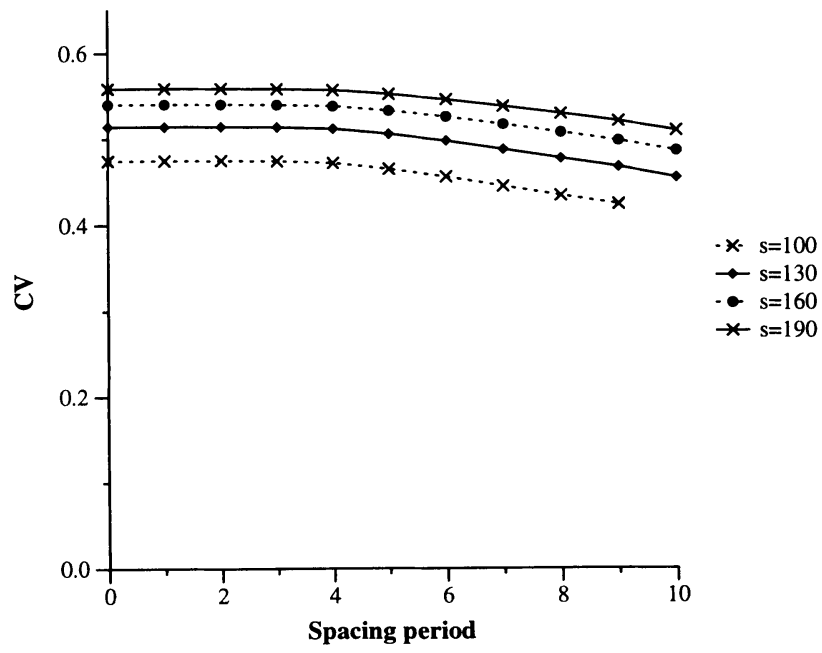

Figure 6: The CV of a tagged VBR-VC as a function of the spacing period, for different values of the silent period $s$ when $d=10$ and the buffer capacity is 10 .

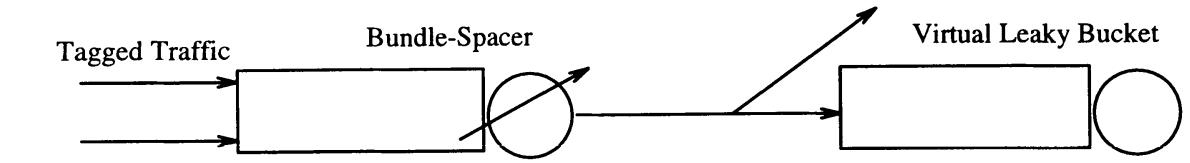

Background Traffic

Figure 7: Loss Probability in the VLB

be more clumped after having passed the bundle-spacer. Moreover, this loss probability is higher when the relative bit rate of the tagged source is higher. However, when the load of the tagged source is maximal (i.e. half of the total load, since otherwise the bundle-spacer behaves as a VC spacer), as in the first example, the required buffer capacity does not exceed 3 , and quickly drops to 2 or even 1 , when $S$ decreases.

\section{(ii) VBR Traffic}

We consider two cases of VBR traffic.

(i) A VBR source with group arrivals. This VBR source is characterized by $\bar{a}^{(1)}=$ ( 0.200 .40 .4$)$. We see that $\lambda_{t}=0.5$. (full line in Figure 8).

(ii) A VBR source without group arrivals. This VBR source is characterized by $\bar{a}^{(2)}=$ ( $\left.\begin{array}{llll}0 & 0.5 & 0 & 0.5\end{array}\right)$. Again $\lambda_{t}=0.5$. (dotted line in Figure 8).

From this example we see that the more the cells of a VC belonging to a bundle are clumped, the more positive the effect of the bundle-spacer on this individual VC is. The type (ii) traffic is less clumped, and therefore has a more flat loss curve, as shown in 
Figure 8 .

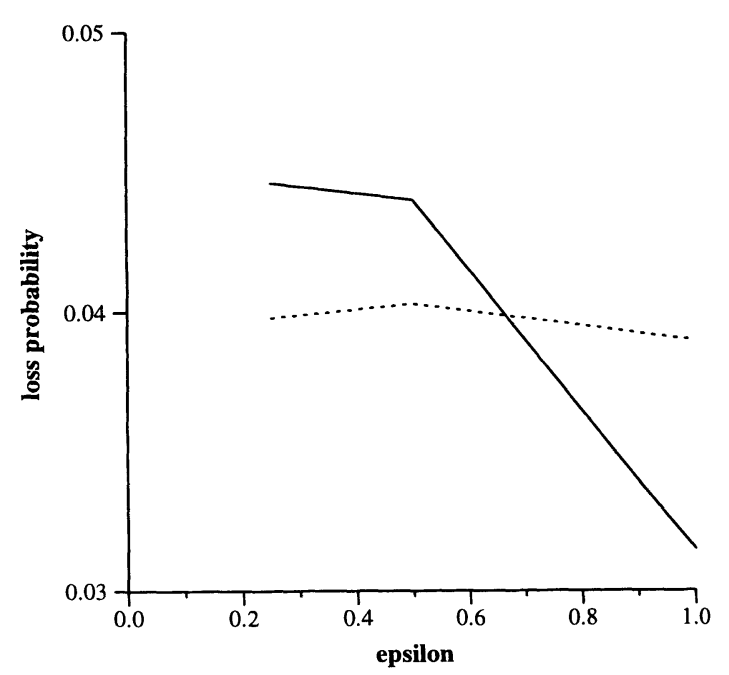

Figure 8: Loss Probability in a VLB, the VBR case

\subsection{Conclusions on output traffic characterisitics}

From the above analysis, we notice that when we tag a VC which belongs to a bundle and study the cell stream of that VC after the cells passed a bundle-spacer, the same behaviour is observed if we either analyze the coefficient of variation or the loss which occurs in a VLB. From the analysis we draw the following conclusions:

1. If the tagged VC is a CBR source (or a VBR source which is close to a CBR source) then increasing the spacing period of a bundle-spacer implies an increase of the frequency of occurrence of clumps of cells. This behaviour is stronger when the bit rate of the tagged source is high with respect to the bit rate of the sum of the other VCs in the bundle. However, from numerical examples it follows that even in extreme circumstances the clumpedness introduced by the spacer is not too high.

2. When the tagged VC is rather bursty, the bundle-spacer decreases the frequency of occurrence of clumps of cells. This is reflected by a lower CV of cells leaving the bundlespacer and a lower loss probability in the VLB, when the spacing period is increased. Moreover, the more the incoming cells are clumped, the more important this effect is. The effect decreases when the relative bit rate of the tagged $\mathrm{VC}$ is low. 


\section{Dimensioning of a Bundle-Spacer for an ATM Ac- cess Network}

In this section we propose a simple rule for dimensioning the spacing period in the bundlespacer occuring in the ATM access network as described in the introduction. This rule takes into account the trade-off between the occurence of clumps of cells in high bit rate connections and the introduction of delay and CDV in the cells stream of low bit rate connections. The traffic behavior at the output of the bundle-spacer is analysed to see how connections with various bit rates are affected by the use of the bundle-spacer dimensioned according to this rule.

\subsection{BPR: A simple rule for dimensioning the spacing period}

In order to take into account the trade-off previously described we propose to dimension the spacing period $\left(T_{s p}\right)$ according to the following rule, called the Bounded Period Rule $(\mathrm{BPR})$

$$
T_{s p}=\min \left(\epsilon T_{t o t}, T_{\max }\right)
$$

with $0<\epsilon \leq 1, T_{\max }>0$ and $T_{\text {tot }}=1 / \lambda_{V P}$.

This rule aims at having for high bit rate connections (small $T_{\text {tot }}$ ) an almost perfect spacing while avoiding for low bit rate connections (large $T_{t o t}$ ) high values of delay and CDV.

Choosing a low value of $T_{\max }$ improves the performance of the bundle-spacer on low bit rate connections, as the delay and CDV introduced by the spacer is small. However, for connections with period $T$ slightly higher than $T_{\max }$ it can produce sequences of cells spaced by $T_{\max }$ instead of $T$, i.e., low values of $T_{\max }$ could lead to clumps of cells and hence damage the QoS of other users.

Dimensioning the bundle-spacer operating according to the BPR means to use values for $\epsilon$ and $T_{\max }$ such that the bundle-spacer behaves satisfactory for all possible bit rates. In what follows we give methods to evaluate how the connections are influenced by the choice of $\epsilon$ and $T_{\text {max }}$.

\subsection{CDV at the output of the bundle-spacer using BPR}

In this analysis we restrict ourselves to CDV as the waiting time introduced by the bundlespacer has been investigated in [3]. We use the following tools:

- A simulator of the access network described in the introduction.

- A simple analytical model for the output traffic of a bundle-spacer isolated from the access network (described in appendix 2).

\subsubsection{CDV when $\epsilon T_{\text {tot }}>T_{\max }$}

In figure 9 we show the 1 -point CDV values $\left(1-10^{-3}\right.$ quantile) for different peak bit rates of a CBR connection multiplexed with either Poisson traffic or a superposition of $64 \mathrm{Kbps}$ CBR sources. There are 80 active NTs and the total load is 0.8 . $T_{\max }$ has been set to 
60 slots. Each NT is equally loaded with a CBR source and background traffic (either 64 Kbps CBR sources or Poisson).

The use of a Poisson process to model the background traffic gives a good estimate for the case of a superposition of $64 \mathrm{Kbps}$ with a high bit rate connection but is too pessimistic when multiplexed with a low bit rate connection (as expected).

From the figure we observe that we can reach values of 1-point CDV below $450 \mu$ secs.

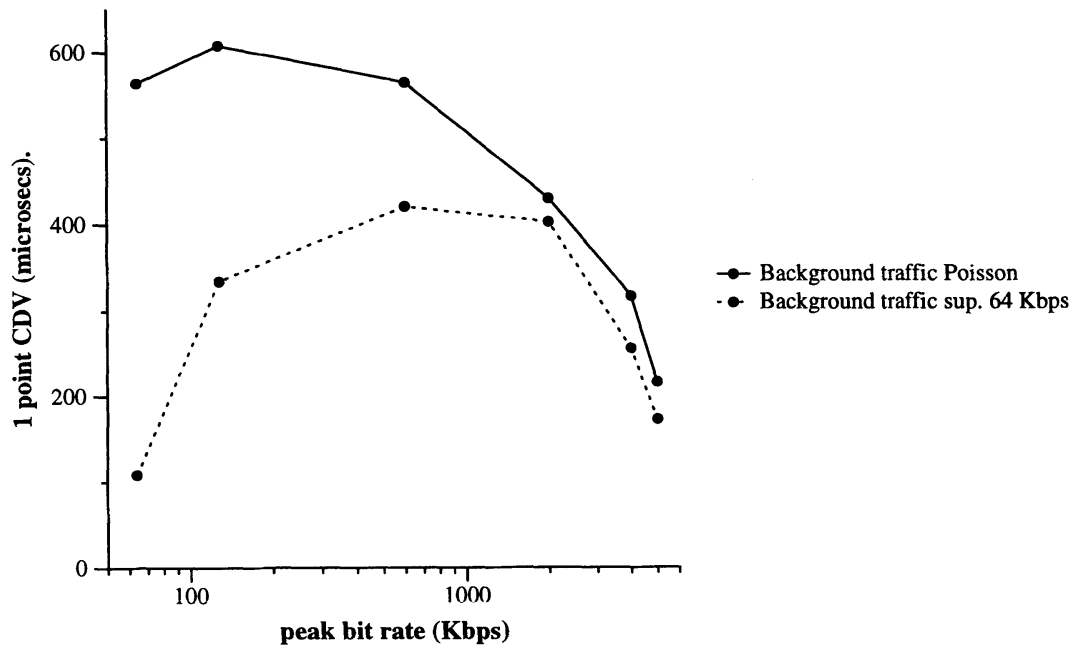

Figure 9:

In figure 10 we show the 1 -point $\mathrm{CDV}$ values $\left(1-10^{-3}\right.$ quantile) for different peak bit rates when varying $T_{\max }$, modelling the background traffic as a Poisson process. We can observe how the 1-point CDV increases with $T_{\max }$. The curves have a "knee" which in this case is around 40 slots. This "knee" behaviour is very important to find an optimum value of $T_{\max }$.

The CDV has a direct impact on the dimensioning of the UPC function in the network. If we want to choose the parameter values of a UPC mechanism like the Leaky-Bucket to control the peak bit rate of the connections, we need a number of credits $N$ that can be estimated as [5]:

$$
N=\left[\frac{\tau}{T}\right]+1
$$

where $\tau$ is a given quantile of the transfer delay of a cell and $\mathrm{T}$ is the period of the CBR connection. Defining $\tau$ as the quantile of the interdeparture time of the cells of a given connection would give similar results (see [4], [6])

Using this formula we obtain the following values of $N$ for the $1-10^{-3}$ quantile (For 


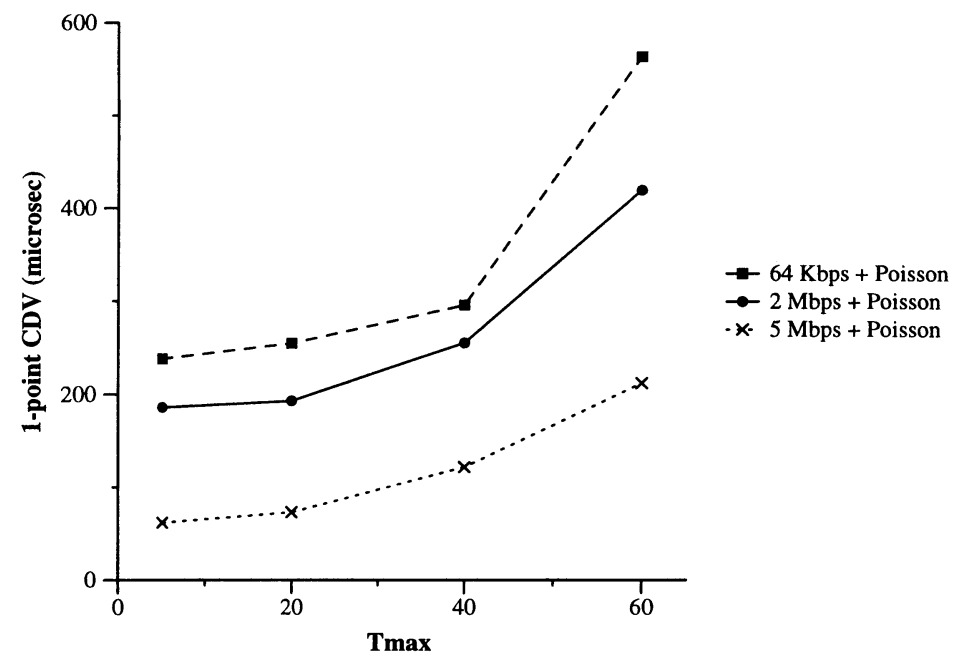

Figure 10:

the case of having CBR background traffic and $T_{\max }=50$ slots).

\begin{tabular}{|l|l|l|}
\hline CBR & $\tau(\mu$ secs $)$ & $\mathrm{N}$ \\
\hline $64 \mathrm{Kbps}$ & 108.0 & 1 \\
$128 \mathrm{Kbps}$ & 334.1 & 1 \\
$600 \mathrm{Kbps}$ & 420.5 & 1 \\
$2 \mathrm{Mbps}$ & 402.5 & 3 \\
$4 \mathrm{Mbps}$ & 255.6 & 4 \\
$5 \mathrm{Mbps}$ & 172.1 & 3 \\
\hline
\end{tabular}

In the case of having connections of several Mbps we can use the model of appendix 2 to estimate the $1-10^{-10}$ quantile of the interdeparture times, obtaining the following results (now we use a Poisson process to model the background traffic and $T_{\max }=50$ slots).

\begin{tabular}{|l|l|l|}
\hline CBR & $\tau(\mu$ secs $)$ & $\mathrm{N}$ \\
\hline $4 \mathrm{Mbps}$ & 462 & 6 \\
$5 \mathrm{Mbps}$ & 378 & 6 \\
$5.5 \mathrm{Mbps}$ & 336 & 6 \\
\hline
\end{tabular}

\subsubsection{CDV when $\epsilon T_{\text {tot }}<T_{\max }$}

Let us study now the case of an NT loaded with a CBR connection multiplexed with Poisson traffic, so that the total traffic in each of the 8 active NTs is $60 \mathrm{Mbps}$. In the 
following table we show the values of $N$ obtained for different values of $\epsilon$ :

\begin{tabular}{|l|l|l|l|}
\hline CBR & $\mathrm{N}(\epsilon=1)$ & $\mathrm{N}(\epsilon=0.9)$ & $\mathrm{N}(\epsilon=0.8)$ \\
\hline $30 \mathrm{Mbps}$ & 8 & 7 & 7 \\
$40 \mathrm{Mbps}$ & 9 & 8 & 7 \\
$50 \mathrm{Mbps}$ & 8 & 7 & 7 \\
\hline
\end{tabular}

\subsection{Efficiency of the bundle-spacer using BPR}

Introducing a certain tolerance in the value of $T_{s p}$ has the drawback that the cells of a given connection at the output of the access network are not spaced anymore according to their declared peak bit rate. To choose a value of $T_{\max }$ and $\epsilon$ we should evaluate how this phenomenon influences the QoS seen by the other users of the network. To do that we simulate a multiplexer which is loaded with the output of a certain number of access networks as the one described in the introduction. We consider two cases: in the first case the customers of the access networks send traffic conforming to their contract (CBR traffic); in the second case the customers send Worst Case Traffic (i.e. bursts of consecutive cells).

The evaluation of the efficiency of the bundle-spacer is made using the following tools:

- A simulator of a multiplexer located after the access network. The input traffic to this simulator can be either gencrated with the model explained in appendix 2 (case $\left.\epsilon T_{\text {tot }}>T_{\max }\right)$ or generated with the results of the simulator of the access network (case $\epsilon T_{\text {tot }}<T_{\max }$ ).

- Analytical models of GEO/D/1 and nD/D/1 queues.

\subsubsection{Case of well behaving customers}

In figures 11 and 12 we show the complementary queue length distribution of a multiplexer located after the access network. The behaviour of the output of an access network is modelled using either the results of appendix 2 or the results from the simulator of the access network.

We have done the following experiment: A high bit rate CBR connection (with period $T=15$ ) is multiplexed with Poisson traffic. After passing through the access network, the original CBR traffic (which has been jitterized by the access network) is multiplexed together with the output traffic of 11 other similar access networks equally loaded. The results of this experiment are shown for different values of $T_{t o t} . T_{\max }=50 \mathrm{slots}$, and $\epsilon=1$. For comparison we have also included the results of a GEO/D/1 queue for the same load (12 geometric sources with a mean period of 15 slots) and of an $\mathrm{nD} / \mathrm{D} / 1$ queue (12 periodic sources with period 15 ).

A similar experiment has been performed to obtain figure 12. In that case we have 120 CBR connections with a period of 150 which have also been jitterized by an access network.

In all cases the output of the access network shows a behaviour similar to an ideal CBR traffic and is always better than the geometric case. 


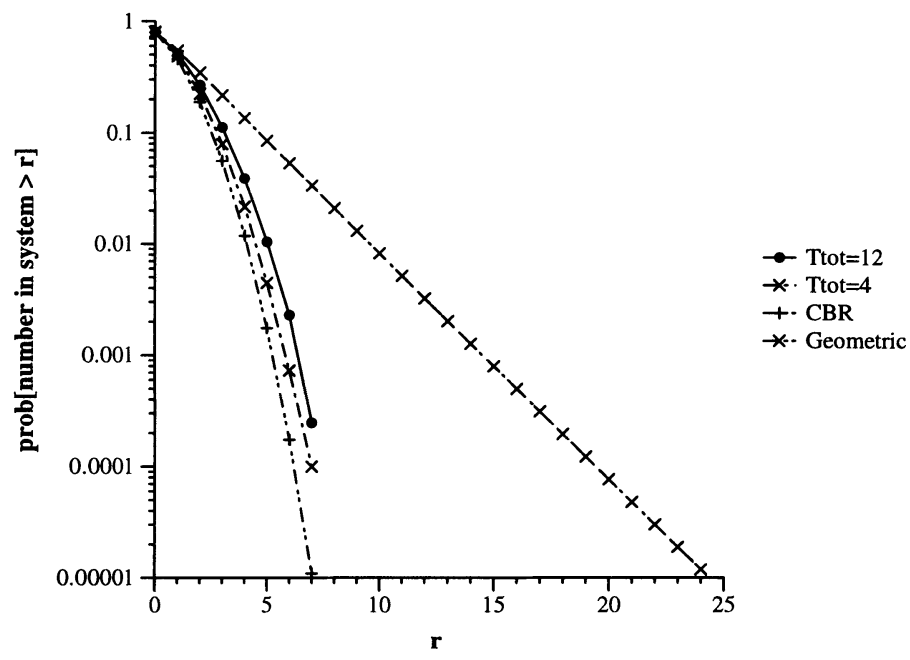

Figure 11:

\subsubsection{Case of WCT customers}

In order to study how the bundle-spacer protects the network against misbehaving customers, we do the following experiment:

We assume that a user declares a peak emission pcriod of value $T$ at connection set-up. However, the user actually sends a Worst Case Traffic (WCT), consisting of $b$ back-to-back cells followed by a silent period, so that the mean bit rate corresponds to the declared peak bit rate $(1 / T)$. If the UPC mechanism has a tolerance of $b$ cells, the traffic can pass transparently through it [1]. Assuming a worst case situation, this traffic is just altered by the bundle-spacer, which spaces the cells a distance $T_{s p}$. To evaluate the efficiency of the bundle-spacer, we study a multiplexer where the input flow is the output of several access networks loaded with WCT sources.

In figure 13 , the declared peak emission period is $T=15$ slots and $T_{t o t}=15$. This corresponds to a high bit rate connection, which can damage more seriously the QoS of the other users of the network, assuming that misbehaving users send WCT with $b=8$.

The value of $\epsilon$ is first chosen so that $T_{s p}=\epsilon T_{t o t}=12$ (i.e. $\epsilon=0.8$, a rather low value). The output of 12 access networks with misbehaving customers are multiplexed together and therefore the multiplexer after the access network has a load of 0.8 . From the simulation results, we can see that the multiplexer distribution is almost not different from the ideal case of $12 \mathrm{CBR}$ connections with $T=15$.

We then perform a second experiment, fixing now $\epsilon=0.667$. In that case the effects of the misbehaving users are already considerable, but always below the geometric hypothesis.

Although not shown here, similar experiments performed for low bit rate connections demonstrate that a value of $T_{\max }$ larger than 40 slots provides a good protection to the 


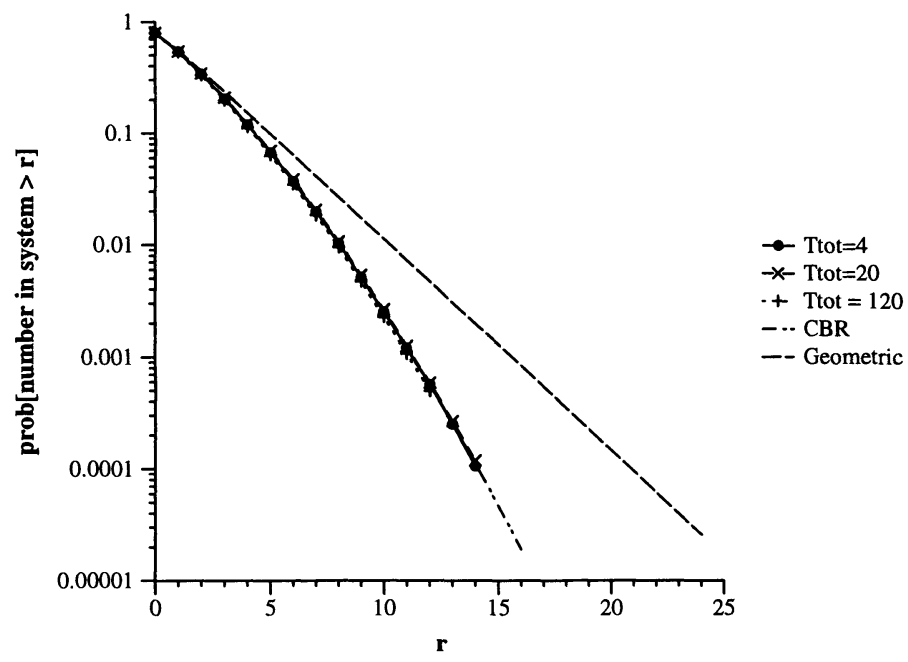

Figure 12:

network.

From the evaluation done we can conclude that a value of $T_{\max }$ around 50 slots, and of $\epsilon$ around 0.8 gives a good trade-off.

\section{Conclusions}

We have proposed and analyzed a traffic shaping function for situations where no VC information is available. This function realizes a spacing of all cell belonging to a bundle of VCs (hence the name bundle-spacer). Although the concept of a bundle-spacer may be applied in various areas, we have focused on the particular case of its use in the access control of an ATM network, where cells of all the VCs carried by the same T-interface are spaced.

The main problem considered in this paper is the dimensioning of the bundle-spacer, i.e. determine a value for the spacing period, based on a trade-off between

(i) the introduction of additional transfer delay and CDV for low bit rate connections

(ii) the avoidance of clumps of cells for high bit rate connections.

A simple rule, called the Bounded Period Rule (BPR), has been proposed and it has been shown using various performance measures, that this rule turns the bundle-spacer into an effective and efficient traffic shaping device.

We believe that also for other applications, the bundle-spacer could offer a cost effective alternative for traffic shaping. In particular in a VP-based ATM network, a VP-spacer could enforce a VP-peak rate before the traffic enters the UPC function. Clearly for these applications of the use of a bundle-spacer, more detailed studies are needed. 


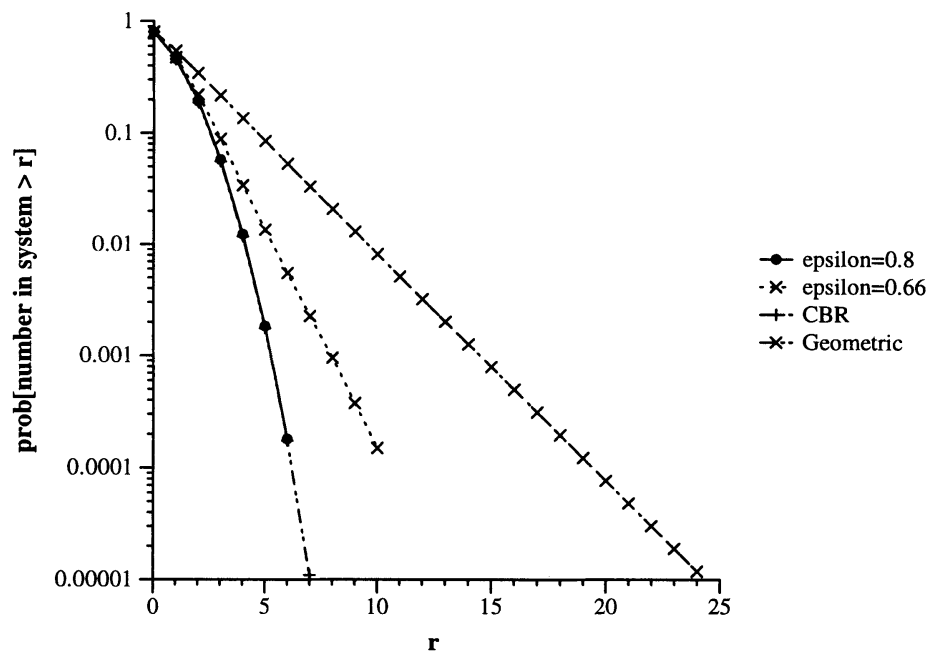

Figure 13:

\section{References}

[1] P.E. Boyer, F.M. Guillemin, M.J. Servel and J-P. Coudreuse Spacing Cells Protects and Enhances Utilization of ATM Network Links, IEEE Network Magazine, Vol. 6, No. 5, September 1992.

[2] E. Wallmeier and T. Woster, A Cell Spacing and Policing Device for Multiple Virtual Connections on one ATM Pipe, Proceedings of the RACE R1022 Workshop on ATM Network Planning and Evolution, London, 1991

[3] O. Casals, J. Garcia and C. Blondia, A Medium Access Control Protocol for an ATM Access Network, Proceedings of the Fifth International Conference on Data Communication Systems and their Performance, High Speed Networks, Eds. H.Perros and Y.Viniotis, Raleigh, North Carolina, USA, October 1993.

[4] Interim Report on CDV models. COST 242

[5] G. Niestegge The Leaky Bucket Policing Method in ATM Networks, Int. Journal of Digital and Analog Communication Systems. Vol. 3, pp. 187-197, 1990.

[6] J. Roberts, F. Guillemin, Jitter in ATM network and its impact on peak rate enforcement, Performance Evaluation, Special Issue on Modelling of High Speed Telecommunication Systems, 1992.

[7] D.A. Stanford and W. Fischer The Interdeparture-Time Distribution for each class in the $\sum_{i} M_{i} / G_{i} / 1$ Queue, Queueing Systems 4, 1989, pp. 179-191. 


\section{Appendix}

\section{Computation of the Coefficient of Variation}

In this appendix we describe the underlying mathematical model which enables us to perform the study, described in section 3.1. For all traffic scenarios the arrival rate of background cells is assumed to be equal to $\lambda$.

Tagged cells arrive according to a batch Poisson process:

Generalising the theory in [7], allowing geometrically distributed batches of cells, we obtain the following expression for the LST of the interdeparture time distribution of tagged cells $(\tau)$ as a function of the spacing period $(m)$ :

$$
\begin{aligned}
\phi_{\tau}(s)= & (1-b) * e^{-m * s} *(A(s) * \\
& \left.\left(1-\phi_{S}(\lambda+\nu-\nu * \eta(s+\lambda))\right)+\phi_{S}(\lambda+\nu-\nu * \eta(s+\lambda)) * \phi_{\beta}(s)\right) .
\end{aligned}
$$

where

$$
\begin{aligned}
\phi_{\beta}(s) & =\frac{\frac{\lambda}{\lambda+\nu+s}+\frac{\nu}{\lambda+\nu+s} * A(s) *\left(e^{-m * s}-\eta(s+\lambda)\right)}{1-\frac{\nu}{\lambda+\nu+s} * \eta(s+\lambda)} \\
A(s) & =\frac{\lambda}{\lambda+\nu-\nu * e^{-m * s}} \\
\phi_{S}(s) & =\frac{s *(1-\rho) *(1-b) * e^{-m s}}{s+\left(\nu-s-\nu * e^{-m * s}\right) * b * e^{-m * s}+(\lambda+\nu) *\left(e^{-m * s}-1\right)} \\
\rho & =(\lambda+\nu) * m .
\end{aligned}
$$

The second moment of the interdeparture time of a tagged cell can now be obtained by differentiating $\phi_{\tau}(s)$ in equation 2 twice and evaluating it at $s=0$. From this result we must subtract the mean interdeparture time of a tagged cell $\left((1-b) / \lambda^{2}\right)$ and divide it by the square of the mean interdeparture time, to obtain the CV.

\section{Tagged cells arrive according to CBR and on/off source :}

To analyze the steady state distribution of the interdeparture time of tagged cells for this traffic scenario, we consider a two dimensional discrete time Markov chain $\left(\# k, R_{k}\right)$. The random variable $\#_{k}$ is the number of cells in the multiplexer while $R_{k}$ represents the remaining service time of the cell in service, both at the $\mathrm{k}$-th slot. Background cells can enter the (queue of the) multiplexer at any moment. However, when a background cell arrives when the server is silent, it has to wait until the end of the slot of arrival before service can start. Finally, tagged cells are assumed to have a loss priority higher than the background cells and will therefore never be lost. When the tagged cell arrives at a totally occupied multiplexer, the last (= background) cell in the queue will be removed and its place will be taken by the tagged cell. We assume that a tagged cell arrives at the system just before a new slot begins. This means that when a tagged cell arrives at the multiplexer at a slot the cell in service planned to leave, the departure of the cell first takes place and then the tagged cell enters the system. When the cell which was about to leave was the only cell in the multiplexer, the tagged cell will start its service immediately after its arrival. For the transition matrix of the Markov chain $\left(\#_{k}, R_{k}\right)$ we consider two situations: a transition to a slot where a tagged cell will arrive (denoted by the matrix $P_{a}$ ) and the transition to a slot where no tagged cell arrives (denoted by the matrix $P$ ). The 
stochastic matrix $P$ can be obtained using straight forward enbedded Markov techniques. In the stochastic matrix $P_{a}$ we read the transition probability from state $\left(\#_{k-1}, R_{k-1}\right)$ to state $\left(\#_{k}, R_{k}\right)$, knowing that at state $\left(\#_{k}, R_{k}\right)$ an arrival of a tagged cell occurs. The transition matrix $P_{a}$ can therefore be obtained from $P$ by the following operations:

$$
\begin{aligned}
P_{a}(0, *) & =0 \\
P_{a}(1,1: m-1) & =0 \\
P_{a}(1, m) & =P(0,0) \\
P_{a}\left(\#_{k}, *\right) & =P\left(\#_{k-1}, *\right), \text { for } 2 \leq k \leq N-1 \\
P_{a}\left(\#_{N}, *\right) & =P\left(\#_{N-1}, *\right)+P\left(\#_{N}, *\right),
\end{aligned}
$$

Denote $P(d)=P^{d-1} * P_{a}$ as the one step transition matrix of having an arrival of a tagged cell at precisely $\mathrm{d}$ slots from now. When we denote the stationary distribution of the Markov chain $\left(\#_{k}, R_{k}\right)$ by $\pi=\pi *(p * P(d)+(1-p) * P(s))$, we obtain the following expression for the distribution of the interdeparture time of tagged cells (for $\mathrm{k}=1, \ldots \max (\mathrm{d}, \mathrm{s})+(\mathrm{N}-1)^{*} \mathrm{~m}, n_{2}=\left\lfloor\left(k-d+\left(n_{1}-1\right) * m+r_{1}\right) / m\right\rfloor+1$ and $r_{2}=$ $\left.k-d+\left(n_{1}-1\right) * m+r_{1}-n_{2} * m\right):$

$$
\begin{aligned}
\operatorname{Pr}\{I D T=k\} & =\sum_{r_{1}=1}^{m} \sum_{n_{1}=2}^{N} \pi\left(n_{1}, r_{1}\right) * \\
& *\left\{p P(d)_{\left\{\left(n_{1}, r_{1}\right) \rightarrow\left(n_{2}, r_{2}\right)\right\}}+(1-p) P(s)_{\left\{\left(n_{1}, r_{1}\right) \rightarrow\left(n s_{2}, r s_{2}\right)\right\}}\right\} \\
& +\pi(1, m) *\left\{p P(d)_{\left(1, r_{1}\right) \rightarrow\left(n_{2}^{*}, r_{2}^{*}\right)}+(1-p) P(s)_{\left(1, r_{1}\right) \rightarrow\left(n s_{2}^{*}, r s_{2}^{*}\right)}\right\} .
\end{aligned}
$$

The squared coefficient of variation can easily be obtained from this equation.

\section{A Model for the Output of the Bundle-Spacer}

Let us assume that a tagged CBR source of period $T$ is multiplexed together with background traffic modelled as a Poisson process. The inverse of the bit rate of the superposition is $T_{t o t}$. A bundle-spacer performs a traffic shaping function, spacing the cells a minimum distance $T_{s p}$. To simplify the model of the traffic of the tagged connection at the output of the bundle-spacer, we assume that the cells arrive to the bundle-spacer without any jitter. If $T_{s p}$ is close to $T_{t o t}$, the aggregate traffic at the output of the bundle-spacer is close to a CBR stream. However a certain jitter (i.e violation of the peak bit rate) can occur over the individual tagged connection.

From the assumptions, we know that the distance between two consecutive arrivals of cells of the tagged source is $T$. If $k$ cells emitted by the background sources arrive during two consecutive cell arrivals of the same connection, and the bundle-spacer is never empty, the two consecutive cells of the tagged sources are sent at a distance $(k+1) T_{s p}$. In fact the bundle-spacer can be empty, but if $T_{s p}$ is close to $T_{t o t}$, the above expression gives a good approximation for the interdeparture time of the tagged CBR connection. Taking into account that we model the background traffic as a Poisson process we obtain the following distribution for $d$, the interdeparture time of the CBR cells:

$$
p\left(d=(k+1) T_{s p}\right)=e^{-\left(\frac{T}{T_{t o t}}-1\right)} \frac{\left(\frac{T}{T_{t o t}}-1\right)^{k}}{k !} .
$$

The above approximation is accurate when $T_{\text {tot }}$ is close to $T_{s p}$. 